\title{
Severity of Infantile Nystagmus Syndrome-Like Ocular Motor Phenotype Is Linked to the Extent of the Underlying Optic Nerve Projection Defect in Zebrafish belladonna Mutant
}

\author{
Sabina P. Huber-Reggi, ${ }^{1,4}$ Chien-Cheng Chen, ${ }^{2,4}$ Lea Grimm, ${ }^{1}$ Dominik Straumann, ${ }^{2,3}$ Stephan C. F. Neuhauss, ${ }^{1,3}$ \\ and Melody Ying-Yu Huang ${ }^{2}$ \\ ${ }^{1}$ Institute of Molecular Life Sciences, University of Zurich, CH-8057 Zurich, Switzerland, ${ }^{2}$ Department of Neurology, University Hospital Zurich, CH-8091 \\ Zurich, Switzerland, ${ }^{3}$ Center for Integrative Human Physiology, University of Zurich, CH-8057 Zurich, Switzerland, and ${ }^{4}$ PhD Program in Integrative \\ Molecular Medicine, Life Science Graduate School, CH-8057 Zurich, Switzerland
}

Infantile nystagmus syndrome (INS), formerly known as congenital nystagmus, is an ocular motor disorder in humans characterized by spontaneous eye oscillations ( $\mathrm{SOs}$ ) and, in several cases, reversed optokinetic response (OKR). Its etiology and pathomechanism is largely unknown, but misrouting of the optic nerve has been observed in some patients. Likewise, optic nerve misrouting, a reversed OKR and SOs with INS-like waveforms are observed in zebrafish belladonna (bel) mutants. We aimed to investigate whether and how misrouting of the optic nerve correlates with the ocular motor behaviors in bel larvae.

OKR and SOs were quantified and subsequently the optic nerve fibers were stained with fluorescent lipophilic dyes. Eye velocity during OKR was reduced in larvae with few misprojecting optic nerve fibers and reversed in larvae with a substantial fraction of misprojecting fibers. All larvae with reversed OKR also displayed SOs. A stronger reversed OKR correlated with more frequent SOs. Since we did not find a correlation between additional retinal defects and ocular motor behavior, we suggest that axon misrouting is in fact origin of INS in the zebrafish animal model. Depending on the ratio between misprojecting ipsilateral and correctly projecting contralateral fibers, the negative feedback loop normally regulating OKR can turn into a positive loop, resulting in an increase in retinal slip. Our data not only give new insights into the etiology of INS but may also be of interest for studies on how the brain deals with and adapts to conflicting inputs.

\section{Introduction}

Infantile nystagmus syndrome (INS) is a congenital ocular motor disorder characterized by involuntary conjugate, predominantly horizontal oscillations of the eyes, present at birth or shortly after (Gresty et al., 1984; Maybodi, 2003). Prevalence is $\sim 2$ per 1000 individuals (Sarvananthan et al., 2009). Visual performance is often impaired affecting occupational and social functioning (Pilling et al., 2005; McLean et al., 2012).

Received Sept. 13, 2012; revised 0ct. 24, 2012; accepted Nov. 1, 2012.

Author contributions: S.P.H.-R., D.S., S.C.F.N., and M.Y.-Y.H. designed research; S.P.H.-R. and L.G. performed research; C.-C.C. contributed unpublished reagents/analytic tools; S.P.H.-R. and S.C.F.N. analyzed data; S.P.H.-R., D.S., S.C.F.N., and M.Y.-Y.H. wrote the paper.

This work was supported by the Zurich Center for Integrative Human Physiology and by the Swiss National Foundation (Grants PMPDP3 139754 and 31003A-118069). We would like to thank Dr. Christian Grimm and Dr. Maarten Frens for fruitful discussion, Dr. Matthias Gesemann for critical reading of this manuscript, and Fabian Huber for help with writing of the analysis software. We thank Kara Dannenhauer for excellent fish care. Imaging was performed with equipment maintained by the Center for Microscopy and Image Analysis, University of Zurich.

Correspondence should be addressed to either of the following: Stephan C.F. Neuhauss, Institute of Molecular Life Sciences, University of Zurich, Winterthurerstrasse 190, CH-8057 Zurich, Switzerland, E-mail: stephan.neuhauss@imls.uzh.ch; or Melody Ying-Yu Huang, Neurology Department, University Hospital Zurich, Frauenklinikstrasse 26, CH-8091 Zurich, Switzerland, E-mail: Ying-Yu.Huang@usz.ch.

DOI:10.1523/JNEUROSCI.4378-12.2012

Copyright $\odot 2012$ the authors $\quad 0270-6474 / 12 / 3218079-08 \$ 15.00 / 0$
INS is often associated with visuosensory abnormalities affecting the cornea, lens, retina or optic nerve, such as aniridia, fovea hypoplasia, and misprojections of optic nerve fibers (e.g., in albinism), but can also be idiopathic (Khanna and Dell'Osso, 2006). Because of the broad range of accompanying symptoms, it has been difficult to define the etiology of INS. Most likely different mechanisms can lead to eye oscillations. The lack of a suitable animal model forced researchers to depend on system modeling, leading to varying hypotheses (Abadi, 2002): INS may be a result of a defect in the internal gain calibration of one of the ocular motor subsystems (Harris, 1995; Broomhead et al., 2000; Jacobs and Dell'Osso, 2004) or a consequence of abnormal positive feedback loops caused by neuronal miswiring (Optican and Zee, 1984; Tusa et al., 1992). Recently, mutations in the gene encoding FERM domain-containing 7 protein (FRMD7) have been related to idiopathic forms of INS (Tarpey et al., 2006; Watkins et al., 2012). FRMD7 is involved in neuronal outgrowth and development, suggesting a role in neuronal network formation (Betts-Henderson et al., 2010).

Recently, we showed that the zebrafish mutant belladonna ( $b e l$ ) may be a suitable animal model for INS in the presence of 
optic nerve fiber misprojections (Huang et al., 2006, 2011). In bel homozygous larvae, a variable fraction of retinal ganglion cell (RGC) axons (optic nerve fibers) misroute in the optic chiasm and project to the wrong brain hemisphere. In wildtype (wt) larvae, all optic nerve fibers project contralaterally forming a crossed optic chiasm. In some bel larvae, all axons misproject ipsilaterally leading to achiasmia. Those larvae display a reversed optokinetic response (OKR) (i.e., the eyes move opposite to the visual stimulus) (Neuhauss et al., 1999; Rick et al., 2000), as observed in some human patients (for example, see Halmagyi et al., 1980). In later studies we observed in larvae with a reversed OKR spontaneous eye oscillations (SOs) (Huang et al., 2006) displaying all the major waveforms typical of INS (Huang et al., 2011). Because of the coexistence of achiasmia and reversed OKR, we formulated the hypothesis that axonal misrouting in achiasmatic larvae results in a positive visuo-ocular motor feedback loop, which increases the velocity of a moving visual stimulus on the retina (called retinal slip), thus leading to the observed ocular motor instability in stable visual surround (Rick et al., 2000; Huang et al., 2006). Patients often show misprojection of a portion of optic nerve fibers (Jeffery, 1997) and display complex OKRs
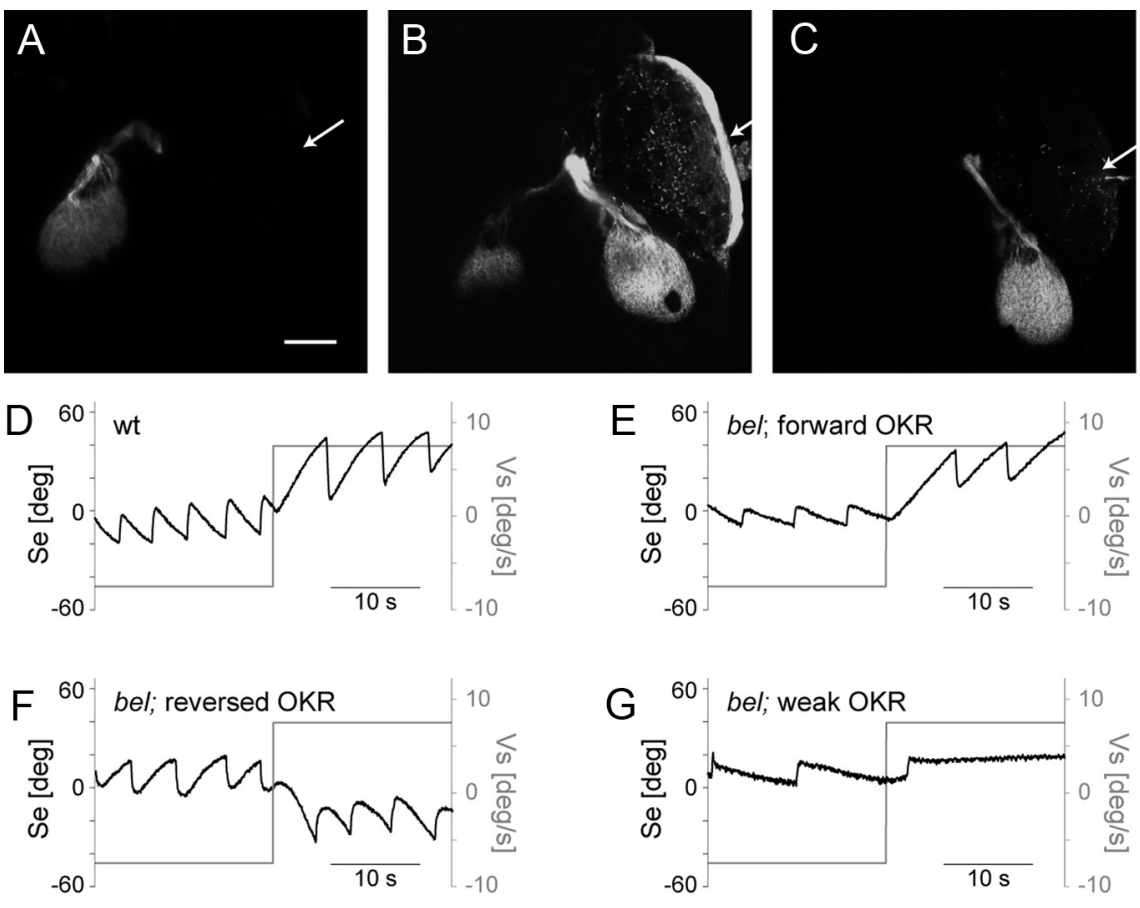

Figure 1. Phenotypes of be/ mutant larvae. A-C, Maximum intensity projections of z-stacks showing projection of optic nerve fibers in be/mutant larvae. The RGC axons were labeled by injecting the green lipophilictracer dye Di0 in the right eye and the red lipophilictracer dye Dil in the left eye. Here the projection from the right eye is shown. Anterior is up. Scale bar, $100 \mu \mathrm{m}$. Arrow points to side of injection. The wt larvae as well as some be/ larvae have a complete contralateral projection ( $A$ ). In some bel larvae a variable fraction of axons misprojects ipsilaterally leading to a bilateral projection (B). Finally, some bel larvae are achiasmatic having a complete ipsilateral projection (O). D-G OKR sample traces of eye position (Se) during stimulation with horizontal moving gratings (Vs). Some bel larvae show a properly directed OKR $(\boldsymbol{E})$. Other larvae show a reversal of the $0 K R(\boldsymbol{F})$. Finally, some larvae show a weaker $0 K R$ with strongly reduced eye velocity and unclear direction (G).

(Collewijn et al., 1985). We speculate that the proportions of correct and incorrect optic nerve fiber projections could be a key factor in determining the ocular motor phenotype in each individual. In this study we quantify for the first time the correlation between extent of optic nerve misprojection and different ocular motor phenotypes in bel mutants.

\section{Materials and Methods}

Fish maintenance and breeding. Fish were maintained and bred as previously described (Mullins et al., 1994). Embryos were raised at $28^{\circ} \mathrm{C}$ in E3 medium $\left(5 \mathrm{~mm} \mathrm{NaCl}, 0.17 \mathrm{~mm} \mathrm{KCl}, 0.33 \mathrm{mM} \mathrm{CaCl}_{2}\right.$, and $0.33 \mathrm{~mm}$ $\mathrm{MgSO}_{4}$ ) and staged according to development in days postfertilization (dpf). bel ( $\left.b_{t} l_{t v 42}\right)$ homozygous larvae were obtained from mating of identified heterozygous carriers. Larvae at $4 \mathrm{dpf}$ were anesthetized with $200 \mathrm{mg} / \mathrm{L} 3$-aminobenzoic acid ethyl esther methane sulfonate (MS-322; Sigma-Aldrich) and sorted according to eye pigmentation phenotype. albino larvae were obtained from mating of homozygous adult fishes.

OKR and SO stimulation. The OKR, a compensatory ocular motor reflex evoked by a moving visual environment, was elicited in a similar way as described previously (Rinner et al., 2005; Huang et al., 2006). Briefly, larvae were embedded dorsal-up in the center of a $35 \mathrm{~mm}$ diameter Petri dish containing prewarmed $\left(28^{\circ} \mathrm{C}\right) 3 \%$ methylcellulose to constrain whole-body movement without significantly affecting eye movement. Using an LCD projector (PLV-Z3000; Sanyo), a computergenerated visual stimulus was projected via a wide-angle conversion lens and a mirror to the internal walls of a paper drum (diameter $=9 \mathrm{~cm}$ ), mounted on a transparent glass plate. The embedded larva was placed in the center of the drum and was illuminated from below with infraredemitting diodes $\left(\lambda_{\text {peak }}=940 \mathrm{~nm}\right.$, BL0106-15-28; Kingbright). If not indicated otherwise, only one eye of the larva was stimulated via restriction of the visual field to the stimulated eye. OKR was elicited with a computer-generated (Straw, 2008) black and white sine-wave grating pattern with $85 \%$ contrast (maximum illumination 400 lux), a spatial frequency of 20 cycles $/ 360^{\circ}$ and an angular velocity of $7.5 \mathrm{deg} / \mathrm{s}$. Stimulation lasted for $180 \mathrm{~s}$ whereby the direction of the moving grating changed every $60 \mathrm{~s}$. The first $60 \mathrm{~s}$, during which OKR is building up, were not used for analysis. For SOs stimulation, one eye was exposed during 5 min with black and white stationary gratings with the same contrast and spatial frequency as described above.

Eye movement recording and analysis. During visual stimulation, binocular eye movements were recorded by an infrared-sensitive CCD camera (Guppy F-038B NIR; Allied Vision Technologies). Frames were processed simultaneously by custom-developed software based on LabView 2009 and NI Vision Development Module 2009 (National Instruments) with a sample rate of 25 samples/s. Data were analyzed by custom-developed software written in MATLAB (MathWorks). Eye position traces were smoothed using a Gaussian filter with cutoff frequency of 2.28 or $2.56 \mathrm{~Hz}$. OKR during unidirectional visual stimulation is characterized by a nystagmus consisting of compensatory slow phases, normally in the direction of the stimulus, and fast phases, or saccades, in the opposite direction bringing the eyes back to a more central position. To distinguish fast phases and slow phases, the eye position traces were split in segments spanning from one change in velocity direction to the next. Subsequently, an eye acceleration threshold was set $\left(18 \% \mathrm{~s}^{2}\right)$. Segments with accelerations lower than this threshold were considered as slow phases. Erroneously classified slow phases were discarded by visual inspection. Slow phases were used for OKR velocity calculation. The OKR velocity was defined as the slow-phase velocity (SPV), computed by averaging the median eye velocity across all slow phases in one measurement. SPVs in the same direction as the stimulus were given positive velocity values; SPVs in the opposite direction were given negative velocity values. Analysis was restricted to the eye with the higher absolute velocity.

Optomotor response stimulation. Optomotor response (OMR), a reflexive swimming behavior in the direction of perceived motion, was measured in a 
similar way as published previously (Neuhauss et al., 1999; Roeser and Baier, 2003). Briefly, $7 \mathrm{dpf}$ larvae were individually transferred into $25 \mathrm{~cm}$ long and $1 \mathrm{~cm}$ wide transparent Plexiglas tanks on an upward-facing monitor. The sidewalls of the tanks were obscured with a matt black adhesive film to avoid reflection of the stimulus. Fish were placed in the middle of the tank and a computer-generated (Straw, 2008) moving sine-wave black and white grating pattern (98\% contrast with maximum illumination of 90 lux, spatial frequency of 4 cycles/1280 pixel (screen size), temporal frequency of $2 \mathrm{~Hz}$ ) was presented twice for $45 \mathrm{~s}$ with an interstimulus interval of $20 \mathrm{~s}$. Fish position over time was recorded by a video camera (HDR-CX130E; Sony). The distance from the starting position to the final position was measured and normalized to the maximal value possible (length of the tank/2). This gave a score between 0 and 1 . Larvae resting at the starting position or swimming in the opposite direction were given a score of 0 . Fish underwent 10 trials with alternating direction of movement. The scores were averaged for all the trials resulting in the optomotor index (OMI).

Electroretinogram. Electroretinograms (ERGs) were recorded as described previously (Makhankov et al., 2004). Briefly, larvae were dark adapted for 30 min. For recording, a reference electrode was placed on a sponge soaked with E3 medium. The larva was placed dorsal-up on a moist paper covering the reference electrode. The recording electrode with a tip diameter of $20 \mu \mathrm{m}$ was filled with E3 and placed on the cornea of the larva. Light stimuli of 100 $\mathrm{ms}$ with interstimulus intervals of $10 \mathrm{~s}$ were applied. The light stimulus intensity was 700 lux.

Anterograde labeling of the optic nerve fibers. To label optic nerve fibers, larvae were fixed in $4 \%$ paraformaldehyde in PBS overnight. For lipophilic dye injection, the fish were embedded dorsal-up in 1.5\% low melting agarose (Nu Sieve GTG Agarose; Lonza) in PBS on a glass slide. Solutions ( $1 \%$ in chloroform) of $\mathrm{DiO}$ (Invitrogen) and DiI (Invitrogen) were pressure injected (40 psi, 20-30 ms pulse time) with a pneumatic Pico Pump (PV820; World Precision Instruments) between lens and retina using glass capillaries. Microscopy $z$-stacks images were obtained using a Leica SP5 confocal laser scanning microscope (Leica Microsystems). Signal intensities were measured using ImageJ (MacBiophotonics).

Statistical analysis. Statistical analysis and graph generation were performed with SPSS Statistics 19 (IBM).

\section{Results}

The bel mutant was first identified in a large-scale screening of mutations affecting optic nerve fiber pathfinding (Karlstrom et al., 1996). It carries a recessive mutation in the zebrafish lhx2 homolog, a Lim domain homeobox transcription factor involved in neural development, including midline axon guidance and eye morphogenesis (Seth et al., 2006). bel mutants show variable morphological eye defects, optic nerve fiber misprojections, and an abnormal pigmentation near the lens, which causes the pupil to appear enlarged (Karlstrom et al., 1996; Seth et al., 2006).

\section{The optic nerve projection and optokinetic phenotypes in bel mutants}

In wt larvae, all optic nerve fibers project contralaterally forming a completely crossed optic chiasm. In bel mutants, the extent of the optic nerve misrouting phenotype is highly variable even within one clutch of eggs. Some bel larvae have contralateral projections like seen in wt (Fig. 1A). The remaining bel larvae display abnormal pathfinding of the optic nerve fibers at the chiasm. Among them, some larvae display a bilateral projection with a subpart of axons growing ipsilaterally (Fig. $1 B$ ), whereas others are achiasmatic, with a completely ipsilateral projection (Fig. 1C) (Rick et al., 2000).

Larvae with contralateral projections show an OKR that is slightly reduced compared with wt but properly directed (forward OKR) (Fig. 1D,E). Larvae with achiasmatic projections show a reversal of the OKR (Fig. 1F) (Rick et al., 2000). Here, we additionally found some homozygous bel larvae with a clearly weaker (in some cases nearly abolished) OKR in terms of magnitude of eye velocity compared with other bel larvae (Fig. 1G). The magnitude of eye velocity refers to the absolute velocity of slow phases, i.e., the velocity independent of the direction.

A weak OKR is often associated with alterations in the retina in both humans and zebrafish (Brockerhoff et al., 1995; Neuhauss et al., 1999; Shin et al., 2006; Wester et al., 2007). Thus, the weak OKR observed in some bel larvae could be caused by morphological defects in the bel eye. On the other hand, it is conceivable that a weak OKR is the consequence of partial misrouting of the optic nerve. Misrouting of a fraction of optic nerve fibers could reduce OKR performance by introducing erroneously interpreted signals in the ocular motor system.

\section{Larvae with a bilateral optic nerve projection display a weak OKR}

To investigate whether a weaker OKR in terms of magnitude of eye velocity, as observed in a subset of bel larvae (Fig. $1 G$ ), is linked to a particular form of optic nerve misrouting, we measured the OKR behavior using a monocular stimulation paradigm and subsequently stained with lipophilic tracer dyes the optic nerve fibers arising from the stimulated eye. Larvae were grouped according to their optic nerve projection phenotype (contralateral, bilateral, or ipsilateral projection) and the OKR SPV was compared among pheno-

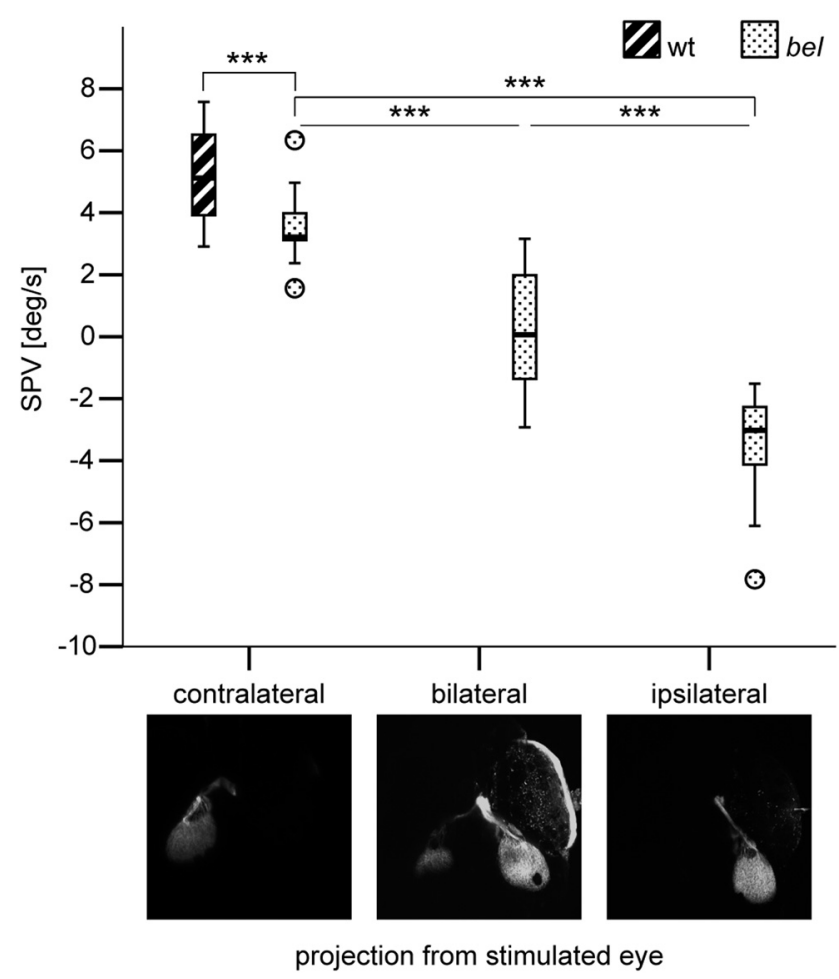

Figure 2. OKR behavior in larvae with different optic nerve fiber projection phenotypes. Box-and-whisker plot of the SPV during monocular OKR stimulation with moving gratings (7.5 $\mathrm{deg} / \mathrm{s})$ in wt $(n=17)$ and in bel larvae with different projection phenotypes ( $n=17$ in each group). A negative SPV value represents reversal of the OKR direction. The ends of the whiskers represent the lowest data point within 1.5 interquartile range of the lower quartile and the highest data point within 1.5 interquartile range of the upper quartile. Circles represent outliers. A one-way ANOVA showed that the OKR behavior differed significantly among groups of bel larvae with different projection phenotypes $\left(F_{(2,48)}=81.007, p<0.001\right)$. Post hoc analysis using the Dunnett's T3 multiple-comparison criterion for significance indicated that the average SPV was significantly closer to 0 in bel larvae with bilateral projections $(M=0.22, S D=1.95)$ than in both bel larvae with contralateral $(M=3.56, S D=1.13)$ and ipsilateral $(M=-3.49$, SD $=1.66)$ projections. ${ }^{* *} p<0.001$. 
types (Fig. 2). As expected from previous reports (Neuhauss et al., 1999; Rick et al., 2000), larvae with a contralateral projection displayed a forward OKR with a slightly reduced velocity, while achiasmatic larvae displayed a reversed OKR. Interestingly, larvae with bilateral projections displayed a significantly weaker OKR compared with the other groups, i.e., a lower magnitude of eye velocity (Dunnett's T3 multiple comparison, $p<0.001)$.

\section{The amount of misprojection correlates with the OKR SPV}

Since the SPV in larvae with bilateral projections showed a high within-group variability (Fig. 2), we asked whether the SPV correlates with the fraction of misprojecting axons. Therefore, the extent of misprojection from the stimulated eye was estimated in each larva by signal intensity quantification on both brain hemispheres and was correlated to the SPV (Fig. 3A). The extent of misprojection exhibited a strong negative correlation with SPV $\left(R^{2}\right.$ $\left.=0.75 ; F_{(1,19)}=56.95, p<0.001\right)$, which was maximal and positive in larvae with no misprojection, reduced in larvae with few misprojecting optic nerve fibers, and negative in larvae with a substantial fraction of misprojecting fibers (Fig. 3B).

In most larvae the amount of misprojection from the two eyes was comparable.

In rare cases misprojection was different between the two eyes (Table 1). In these larvae we compared the correlation between the fraction of misprojecting fibers and SPV separately for each eye being stimulated. Within the same subject, the SPV correlated with the projection phenotype of the stimulated eye. This was particularly evident for one specimen (larva 4 in Table 1) in which the projection from the right eye was contralateral and the projection from the left eye was ipsilateral. Thus axons from both eyes projected to the same brain hemisphere. Stimulation of the right eye led to a positive SPV $(+3.07 \mathrm{deg} / \mathrm{s})$, stimulation of the left eye led to nearly the same absolute value, but negative $(-3.08$ deg/s) (Table 1).

\section{Correlation between $\mathrm{OKR}$ velocity and $\mathrm{SOs}$}

INS patients show SOs of the eyes despite absent movement of the visual surround (Gresty et al., 1984). We previously reported about SOs in the presence of stationary gratings in a subset of bel larvae with a reversed OKR and presumed achiasmia (Huang et al., 2006). Here, we correlate the SOs with a quantitative extent of optic nerve fiber misprojection.

OKR in wt larvae ceases with the termination of motion of the visual surround and subsequently only saccades and small eye drifts are observed (Fig. $4 A$ ). In larvae with SOs the eyes keep moving under stationary structured background with eye position traces displaying characteristic nystagmus waveforms that can easily be distinguished from the OKR (Fig. 4B) (Huang et al., 2011). We hypothesized that both reversed OKR and SOs may be caused by optic nerve fiber misrouting. To compare the OKR phenotype with the occurrence of SOs, we first quantified the OKR SPV during stimulation with a monocular paradigm and
Table 1. SPV in larvae with different optic nerve fibers projection phenotypes from the two eyes

\begin{tabular}{llcc}
\hline & St.eye & \% mispr. & SPV (deg/s) \\
\hline Larva 1 & Right & 91 & -2.19 \\
& Left & 100 & -3.40 \\
Larva 2 & Right & 67 & -0.77 \\
& Left & 94 & -3.49 \\
Larva 3 & Right & 75 & -1.30 \\
& Left & 0 & 1.14 \\
Larva 4 & Right & 0 & 3.07 \\
& Left & 100 & -3.08 \\
\hline
\end{tabular}

St.eye, Stimulated eye; \% mispr., percentage of misprojecting axons from stimulated eye; SPV, slow-phase velocity.

subsequently we quantified the occurrence of SOs during fixation of a stationary background. Figure $4 C$ shows the correlation between the duration of SOs and SPV and between the duration of SOs and the projection phenotype of optic nerve fibers. The wt larvae and bel larvae with a contralateral projection never displayed SOs. We did not observe SOs in larvae with a forward OKR, either. In contrast, both larvae with bilateral and completely ipsilateral projections displayed SOs if their OKR was reversed. The duration of SOs positively correlated with the velocity of the reversed $\operatorname{OKR}\left(R^{2}=0.52 ; F_{(1,23)}=22.56\right.$; $p<0.001)$.

Additional visual defects in bel mutants do not correlate with the ocular motor behavior

Although our data show a very strong correlation between the fraction of misprojecting optic nerve fibers and the ocular motor 

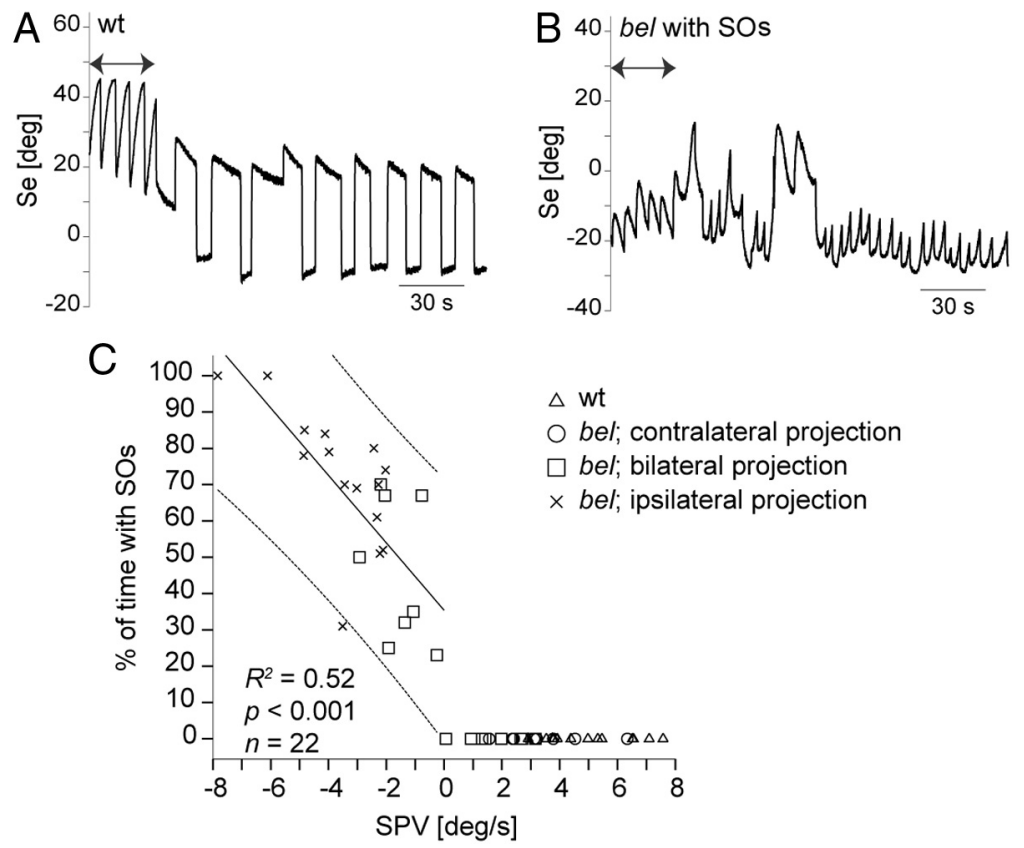

Figure 4. Correlation between velocity of the reversed OKR and occurrence of SOs. A, B, Sample traces of eye position (Se) during stimulation with moving $(7.5 \mathrm{deg} / \mathrm{s})$ and horizontal stationary gratings. Arrows indicate a unidirectionally moving grating. The wtlarva only showed saccades and slow eye drifts during presentation of stationary gratings $(\boldsymbol{A})$. Under identical conditions, be/larvae with a reversal of the OKR displayed SOs without motion stimulus (B).C, Correlation between SPV during monocular OKR stimulation with moving gratings $(7.5 \mathrm{deg} / \mathrm{s}$ ) and occurrence of $\mathrm{SO}$ s. $\mathrm{SO}$ s were elicited during $5 \mathrm{~min}$ by stationary black and white gratings presented to the same eye that was optokinetically stimulated. Finally, the optic nerve fibers from the stimulated eye were stained (see Materials and Methods). The duration of $\mathrm{SO}$ s showed a positive linear correlation with the reversed $0 \mathrm{KR}\left(R^{2}=0.52 ; F_{(1,21)}=22.56, p<0.001\right)$. Line of best fit and individual $95 \%$ confidence interval are shown. Line of best fit was only defined for SPV $<0$, since larvae with SPV $>0$ never displayed SOs.

behavior, they cannot rule out a role of retinal defects in the ocular motor phenotype. Subtle morphological changes-a pigmentation defect and an acellular aggregate near the lens, disorganization of Müller glial cells - have been observed in the bel eye (Seth et al., 2006). Like the optic nerve projection defect, those changes are highly variable. With the correlation studies outlined above we cannot exclude the possibility that the more severe ocular motor phenotype seen in larvae with more misprojecting axons is eventually due to a more severe general eye phenotype. Thus, larvae with more misprojecting axons could as well have more severe morphological defects in the eye. The retinal cell disorganization in bel larvae has been histologically described (Seth et al., 2006). However, there is no established method for quantification of the extent of those defects. Nevertheless, if the observed morphological changes in the eye played a role, we would expect additional aspects of visual performance to be affected and we would see a correlation between them and the ocular motor behavior.

Morphological changes of the Müller glial cells affecting OKR are likely reflected in the electrical activity in the retina (Miller and Dowling, 1970). Therefore, an OKR phenotype caused by disorganization of Müller glial cells is expected to result in an altered overall electrical activity of the retina as measured by the ERG. The ERG output of dark-adapted larvae consists of a small a-wave reflecting photoreceptor activation and a larger b-wave reflecting ON bipolar cell activation (Fig. 5A). Here, we measured the ERG and quantified the b-wave amplitude in bel larvae to find out if there is any functional defect affecting the ON pathway, which is known to be involved in the OKR (Emran et al., 2007). To exclude any correlation between putative functional defects in the outer retina and ocular motor instabilities, we first quantified the OKR SPV of each larva during monocular OKR stimulation and subsequently recorded the ERG from the stimulated eye. Larvae were classified according to the SPV and the b-wave amplitude was compared between groups. The b-wave amplitude was significantly reduced in bel mutants compared with wt (one-way ANOVA, $\left.F_{(5,73)}=5.64 ; p<0.001\right)$. However, we did not find any difference between bel larvae with different SPV (Tukey-HSD, $p>0.05$ ) (Fig. 5B).

These results indicate that, although morphological eye defects in bel mutants do functionally affect the ON pathway, they equally change the retinal electrical activity among bel groups with different ocular motor phenotypes.

Morphological defects in the retina could affect visual acuity and motion vision (Neuhauss et al., 1999; Muto et al., 2005). However, they would affect general motion-dependent vision pathways and not only the OKR. To test whether the OKR abnormalities observed are specific to horizontal motion and thus caused by optic nerve fibers misrouting or whether general motion perception pathways are impaired, we performed an OMR assay. The OMR is a reflexive swimming behavior in the same direction as the perceived motion in the surround and is an indicator for the ability to correctly detect forward motion (Neuhauss et al., 1999; Muto et al., 2005). Of each larva we first measured the SPV during binocular optokinetic stimulation. Then we quantified the OMR (see Materials and Methods) and subsequently stained the retinofugal projections. The wt larvae displayed an average OMI (distance swum/length of the tank) of $0.56(M=$ $0.56, \mathrm{SD}=0.17)$ The magnitude of the response was significantly reduced in bel larvae (one-way ANOVA, $F_{(6,114)}=10.75 ; p<$ 0.001 ), and was comparable to the magnitude in albino mutants (Fig. 5C). albino mutants have defects in the retinal pigment epithelium without major visual impairments (Neuhauss et al., 1999). When a whole clutch of albino larvae was placed inside a tank and the OMR performance of the clutch was analyzed (measured as the percentage of larvae being at the end of the tank after the trial), only a slight reduction in performance was observed compared with wt (data not shown). These results suggest a slight deficiency in perceiving and processing forward motion in bel mutants. Nevertheless, we did not detect any difference in the OMR magnitude between bel larvae with different SPV during OKR (Tukey-HSD, $p>0.05$ ) (Fig. 5C) or different optic nerve projection phenotypes (data not shown), indicating that the general deficit in motion vision does not correlate with the ocular motor abnormalities.

Together, our data indicate that the observed morphological defects in the retina are not related to the INS-like phenotype.

\section{Discussion}

Several studies in humans have described an association of reversed OKR and/or SOs with abnormal decussation of optic nerve fibers in the optic chiasm (St John et al., 1984; Collewijn et al., 1985; McCarty et al., 1992). Often, misrouted axons are mixed 
in with correctly projecting ones (Jeffery, 1997). Possibly because of this, the OKR is not always reversed but can also be weak or nearly normal (Collewijn et al., 1985). In our study we show for the first time in bel mutants a wide range of forward and reversed OKR with different velocities (Fig. 1) and an effect on the ocular motor phenotype when a few optic nerve fibers are misrouted. To understand this effect, we performed an in-depth correlation study between the extent of optic nerve misprojection and the ocular motor phenotype. Our data not only give new insights into the pathomechanisms underlying INS in patients with optic nerve fiber misprojections but are also an example of how interference with feedback loops in the nervous system affects behavior and on how the brain adapts to conflicting neuronal signals.

\section{OKR velocity and direction correlate with the fraction of misprojecting optic nerve fibers}

We show that the OKR efficiency is directly correlated with the extent of optic nerve misrouting with a reduction of the OKR SPV in larvae with few misprojecting fibers and a reversal in larvae with a substantial amount of misprojecting fibers (Fig. 3). We show a direct correlation between optic nerve misrouting and OKR phenotype even in larvae in which the projection phenotype is different between the two eyes (Table 1). These results further support our previously formulated hypothesis that misprojecting optic nerve axons lead to directioninverted interpretation of an information about horizontal whole field motion (Rick et al., 2000). In wt zebrafish a moving visual input triggers movement of the eyes in the same direction via the contralaterally located OKR integration network (OIN; Fig. 6A). This negative feedback loop reduces retinal slip. In achiasmatic larvae the visual input signal is fed to the ipsilaterally located OIN and is therefore interpreted as originating from the opposite eye. A stimulation of the left eye with horizontal clockwise rotating gratings triggers a temporal to nasal movement of the eyes. Since the information is interpreted as originating from the right eye in an achiasmatic larva, temporal to nasal corresponds to counterclockwise rotation so that the eyes will move in the opposite direction (Fig. 6E). This generates a positive feedback loop in the optokinetic system, which further increases retinal slip. In larvae with a bilateral projection, misrouting of a fraction of optic nerve fibers leads to a conflict between correctly and erroneously interpreted signals. Few misprojecting axons might insert some erroneous signals in an otherwise normal OKR, thus just leading to a reduction of the SPV (Fig. 6B). When around half of the axons are misprojecting, the conflict between correct and erroneous signals is the highest leading to a disappearance of the OKR (Fig. 6C). Finally, when a majority of axons project to the wrong brain hemisphere, this misinterpreted information prevails, leading to a reversal of the OKR (Fig. 6D).

Previous studies have shown that pretectal nuclei rather than the tectum, which is the main arborization field of RGCs, are
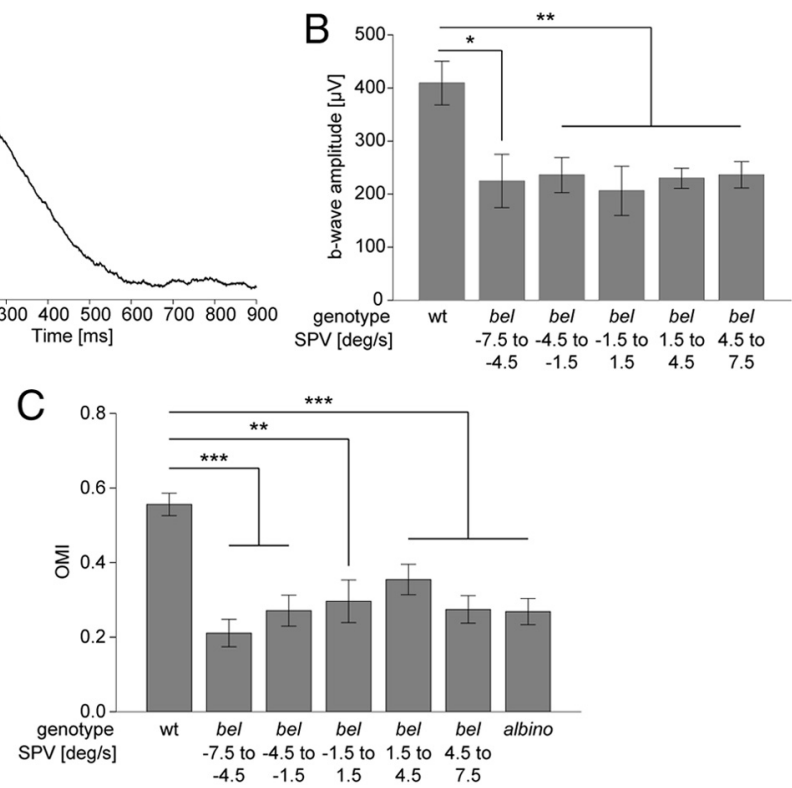

$\begin{array}{lllll}-4.5 & -1.5 & 1.5 & 4.5 & 7.5\end{array}$

Figure 5. General visual properties of bel larvae. $A, B$, Electroretinography. $A, A$ typical ERG trace of a wt larva is shown. A stimulus of 700 lux was used. Arrowhead points to the a-wave; arrow points to the $b$-wave. $\boldsymbol{B}$, Graph showing $b$-wave average significantly from all categories of be/ larvae and from albino mutants. The mean $0 \mathrm{MI}$ did not significantly differ between bel larvae with different OKR phenotypes or between bel and albino larvae (Tukey-HSD, $p>0.05$ ). ${ }^{* *} p<0.01 ;{ }^{* *} p<0.001$.

involved in the zebrafish OKR circuit (Roeser and Baier, 2003). As a consequence, we expect only a small subset of RGCs to be involved in the OKR. Since we stained the complete optic nerve, the correlation between OKR phenotype and extent of misprojection in the general RGC population is surprisingly strong. This can be explained by the fact that terminals of misprojecting axons are found at different arborization locations and topographic arborization of RGC axons is maintained in bel mutants (Karlstrom et al., 1996). Thus misprojecting axons can originate from everywhere in the retina and the amount of misprojection in the whole population reflects quite well the amount of misprojection within the subclass of RGCs involved in the OKR circuit.

\section{Extent of the OKR reversal correlates with the occurrence of spontaneous oscillations}

We could demonstrate that SOs only occur in larvae with a reversed OKR and that the overall duration of oscillations during fixations correlates positively with the reversed SPV. This correlation is highly significant $(p<0.001)$ but rather low $\left(R^{2}=0.52\right)$. This can easily be explained by the fact that, once oscillations stop, a random spontaneous saccade is most likely needed to start oscillations again.

In zebrafish optic nerve fibers pathfinding and arborization are completed before visual experience and development of the eye movements (Burrill and Easter, 1994; Easter and Nicola, 1996), This is also true for misprojecting axons of the optic nerve fibers in bel mutants (Seth et al., 2006). Thus, SOs cannot be the primary cause that leads to adaptation of the visual system. In contrast, the relationships observed agree with the idea of a feed- 
A

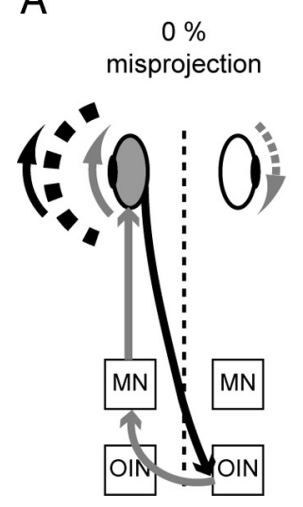

B

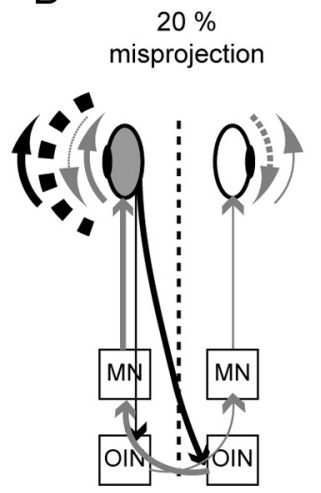

C

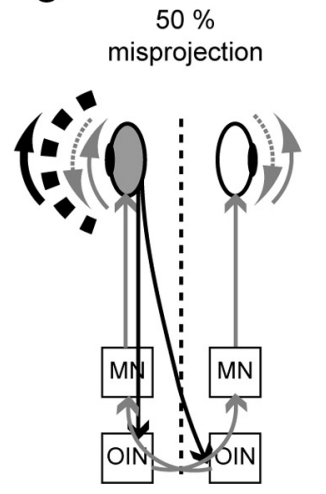

$\mathrm{D}$

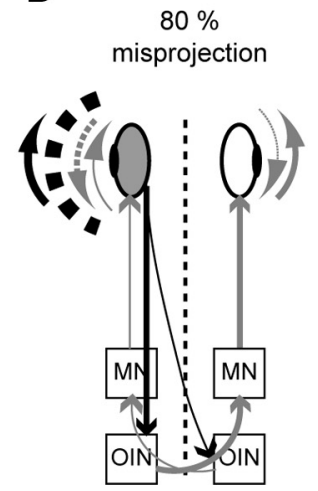

E

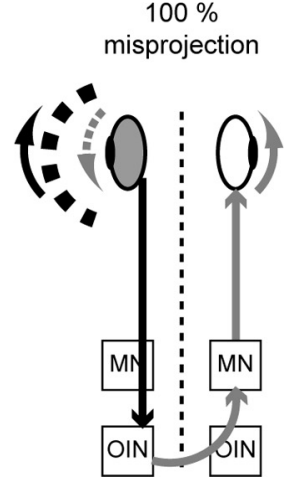

Figure 6. Model of OKR feedback loop in bel larvae with different amounts of misprojection. $A$, In the absence of misprojecting fibers, the clockwise rotating visual stimulus (black and white) is perceived by the stimulated eye (gray) and the information is transferred across the midline (dashed line) to the yet unknown contralaterally located OIN. The 0IN sends the signal again across the midline to the motor nuclei (MN) of the stimulated eye, leading to a compensatory eye movement in the direction of the stimulus (gray arrow around the eye). The other eye is driven in the same direction (dashed arrow). $\boldsymbol{B}-\boldsymbol{E}$, With an increasing amount of misprojection, more signal is sent to the ipsilateral OIN, leading to transmission of the information to the wrong eye (see Discussion). The size of the arrows represents the amount of information flow in each direction.

back loop that has become positive because of the miswired optokinetic nerve fibers, thus leading to the inability to stabilize retinal slip. Misrouting of a small fraction of axons leads to a weaker OKR but not to SOs. Interestingly, the observation of a weak OKR in the absence of SOs has been made also in obligate carriers of the FRMD7 mutation in what can be seen as a subclinical manifestation of idiopathic INS (Thomas et al., 2008).

It has been suggested that the reversal of the OKR is an illusion due to the superimposition of SOs (Yee et al., 1980). We can exclude this in bel mutants because in most cases the OKR had its own characteristics with decelerating slow phases synchronized with the direction of stimulation, either being in the same direction or being inverted (Fig. 1). We only rarely observed superimposition of OKR and SOs. Those few larvae were excluded from analysis.

\section{Additional visual defects in bel mutants do not correlate with the ocular motor behavior}

INS in humans has not only been associated to optic nerve misrouting but also to abnormalities in the retina (Khanna and Dell'Osso, 2006). Subtle retinal defects-a pigmentation defect and an acellular aggregate near the lens, disorganization of Müller glial cells-are also observed in bel mutants (Seth et al., 2006). Comparison between the extent of the pigmentation defect and the ocular motor phenotype did not provide evidence for such a relationship (data not shown). However, a possible correlation and causal relationship between defects in the retina and ocular motor abnormalities cannot be excluded a priori. We previously reported on a slight reduction of eye velocity during OKR in bel larvae with a forward OKR (Huang et al., 2006). Here, we found a significant reduction of the ERG b-wave amplitude, indicative of functional defects in the ON-pathway, and a significant impairment of general motion pathways (Fig. 5). Thus morphological eye defects affect vision in bel larvae, but do not lead to blindness, as indicated by the residual ERG b-wave and by a normal increase in reflexive body movement as a reaction to changes in illumination (data not shown). This is also true for $b e l$ larvae with an abolished OKR. Moreover, general visual impairment is present homogenously in all bel larvae, regardless of the ocular motor phenotype. Therefore, we can assume that INS-like findings in zebrafish are caused by the optic nerve fibers misrouting.
Significance for the debate on the etiology of INS in humans The mechanisms underlying INS are still under debate. Approaches using mathematical models support possibilities that range from an essentially motor defect (Harris, 1995; Broomhead et al., 2000; Jacobs and Dell'Osso, 2004) to a clearly sensorydriven pathology (Optican and Zee, 1984; Tusa et al., 1992). Another hypothesis states that INS is a developmental disorder triggered by any event that disturbs either calibration of visual feedback loops (Tusa et al., 2002; Harris and Berry, 2006a, b; Dell'Osso, 2006) or regulation of innervations during early development (Berg et al., 2012). Here, we show that INS in zebrafish has a sensory origin, being triggered by optic nerve fiber misrouting. The association of INS with visual pathway abnormalities in a subset of human patients (e.g., in albinism) suggests that optic nerve misrouting may indeed trigger the pathology in a similar way in those patients. However, the observation of SOs only in larvae with an OKR reversal is in contrast with reports in albino humans and, most recently, in hypopigmented mice (Collewijn et al., 1985; Traber et al., 2012). Interestingly, in the hypopigmented mouse model the OKR became reversed if the visual stimulation was restricted to the temporal retina only, where most misrouting occurs (Traber et al., 2012). This suggests that a reversal of the OKR could also be elicited in subjects with only a limited fraction of axons misprojecting if only that very part of the retina is stimulated from where those misrouted axons originate. In contrast, a full-field stimulation of the OKR in a laboratory setting, as is now routinely performed, stimulates all optic nerve fibers. Together, our data combined with findings in other models suggest that, during early development, optokinetic stimulation of retinal regions from where misrouting axons originate increases retinal slip and triggers SOs. Adaptive calibration of the efferent ocular motor system later during development may lead to maintenance of SOs-although at reduced amplitude-when few misrouting axons are stimulated or even in darkness, thus in the absence of vision.

\section{Conclusion}

Our study provides detailed insights on how optic nerve misrouting influences ocular motor stability in the zebrafish INS model. The use of larval zebrafish enables us to study the triggering mechanisms without the complication of further adaptive 
mechanisms during the ocular motor system development. We suggest that abnormal decussation of retinofugal fibers at the optic chiasm might be the origin of INS in a substantial number of patients, although not in all as eye oscillations also occur in patients that do not show abnormal decussation. Nevertheless, as visual pathway abnormalities are not routinely investigated in INS patients and are difficult to quantify, they are most likely underdiagnosed.

\section{References}

Abadi RV (2002) Mechanisms underlying nystagmus. J R Soc Med 95:231234. CrossRef Medline

Berg KT, Hunter DG, Bothun ED, Antunes-Foschini R, McLoon LK (2012) Extraocular muscles in patients with infantile nystagmus: adaptations at the effector level. Arch Ophthalmol 130:343-349. CrossRef Medline

Betts-Henderson J, Bartesaghi S, Crosier M, Lindsay S, Chen HL, Salomoni P, Gottlob I, Nicotera P (2010) The nystagmus-associated FRMD7 gene regulates neuronal outgrowth and development. Hum Mol Genet 19: 342-351. CrossRef Medline

Brockerhoff SE, Hurley JB, Janssen-Bienhold U, Neuhauss SC, Driever W, Dowling JE (1995) A behavioral screen for isolating zebrafish mutants with visual system defects. Proc Natl Acad Sci U S A 92:10545-10549. CrossRef Medline

Broomhead DS, Clement RA, Muldoon MR, Whittle JP, Scallan C, Abadi RV (2000) Modelling of congenital nystagmus waveforms produced by saccadic system abnormalities. Biol Cybern 82:391-399. CrossRef Medline

Burrill JD, Easter SS Jr (1994) Development of the retinofugal projections in the embryonic and larval zebrafish (Brachydanio rerio). J Comp Neurol 346:583-600. CrossRef Medline

Collewijn H, Apkarian P, Spekreijse H (1985) The oculomotor behaviour of human albinos. Brain 108:1-28. CrossRef Medline

Dell'Osso LF (2006) Biologically relevant models of infantile nystagmus syndrome: the requirement for behavioral ocular motor system models. Semin Ophthalmol 21:71-77. CrossRef Medline

Easter SS Jr, Nicola GN (1996) The development of vision in the zebrafish (Danio rerio). Dev Biol 180:646-663. CrossRef Medline

Emran F, Rihel J, Adolph AR, Wong KY, Kraves S, Dowling JE (2007) OFF ganglion cells cannot drive the optokinetic reflex in zebrafish. Proc Natl Acad Sci U S A 104:19126-19131. CrossRef Medline

Gresty M, Page N, Barratt H (1984) The differential diagnosis of congenital nystagmus. J Neurol Neurosurg Psychiatry 47:936-942. CrossRef Medline

Halmagyi GM, Gresty MA, Leech J (1980) Reversed optokinetic nystagmus (OKN): mechanism and clinical significance. Ann Neurol 7:429-435. CrossRef Medline

Harris C, Berry D (2006a) A developmental model of infantile nystagmus. Semin Ophthalmol 21:63-69. CrossRef Medline

Harris CM (1995) Problems in modelling congenital nystagmus: towards a new model. In: Eye movement research: processes, mechanisms and applications (Findlay JM, Walker R, Kentridge RW, eds), pp 239-253. Amsterdam: Elsevier.

Harris CM, Berry D (2006b) A distal model of congenital nystagmus as nonlinear adaptive oscillations. Nonlinear Dynamics 44:367-380. CrossRef

Huang MY, Chen CC, Huber-Reggi SP, Neuhauss SC, Straumann D (2011) Comparison of infantile nystagmus syndrome in achiasmatic zebrafish and humans. Ann N Y Acad Sci 1233:285-291. CrossRef Medline

Huang YY, Rinner O, Hedinger P, Liu SC, Neuhauss SC (2006) Oculomotor instabilities in zebrafish mutant belladonna: a behavioral model for congenital nystagmus caused by axonal misrouting. J Neurosci 26: 9873-9880. CrossRef Medline

Jacobs JB, Dell'Osso LF (2004) Congenital nystagmus: hypotheses for its genesis and complex waveforms within a behavioral ocular motor system model. J Vis 4:604-625. CrossRef Medline

Jeffery G (1997) The albino retina: an abnormality that provides insight into normal retinal development. Trends Neurosci 20:165-169. CrossRef Medline

Karlstrom RO, Trowe T, Klostermann S, Baier H, Brand M, Crawford AD, Grunewald B, Haffter P, Hoffmann H, Meyer SU, Müller BK, Richter S, van Eeden FJ, Nüsslein-Volhard C, Bonhoeffer F (1996) Zebrafish mutations affecting retinotectal axon pathfinding. Development 123: 427-438. Medline

Khanna S, Dell'Osso LF (2006) The diagnosis and treatment of infantile nystagmus syndrome (INS). ScientificWorldJournal 6:1385-1397. CrossRef Medline

Makhankov YV, Rinner O, Neuhauss SC (2004) An inexpensive device for non-invasive electroretinography in small aquatic vertebrates. J Neurosci Methods 135:205-210. CrossRef Medline

Maybodi M (2003) Infantile-onset nystagmus. Curr Opin Ophthalmol 14: 276-285. CrossRef Medline

McCarty JW, Demer JL, Hovis LA, Nuwer MR (1992) Ocular motility anomalies in developmental misdirection of the optic chiasm. Am J Ophthalmol 113:86-95. Medline

McLean RJ, Windridge KC, Gottlob I (2012) Living with nystagmus: a qualitative study. Br J Ophthalmol 96:981-986. CrossRef Medline

Miller RF, Dowling JE (1970) Intracellular responses of the Muller (glial) cells of mudpuppy retina: their relation to b-wave of the electroretinogram. J Neurophysiol 33:323-341. Medline

Mullins MC, Hammerschmidt M, Haffter P, Nüsslein-Volhard C (1994) Large-scale mutagenesis in the zebrafish: in search of genes controlling development in a vertebrate. Curr Biol 4:189-202. CrossRef Medline

Muto A, Orger MB, Wehman AM, Smear MC, Kay JN, Page-McCaw PS, Gahtan E, Xiao T, Nevin LM, Gosse NJ, Staub W, Finger-Baier K, Baier H (2005) Forward genetic analysis of visual behavior in zebrafish. PLoS Genet 1:e66. CrossRef Medline

Neuhauss SC, Biehlmaier O, Seeliger MW, Das T, Kohler K, Harris WA, Baier H (1999) Genetic disorders of vision revealed by a behavioral screen of 400 essential loci in zebrafish. J Neurosci 19:8603-8615. Medline

Optican LM, Zee DS (1984) A hypothetical explanation of congenital nystagmus. Biol Cybern 50:119-134. CrossRef Medline

Pilling RF, Thompson JR, Gottlob I (2005) Social and visual function in nystagmus. Br J Ophthalmol 89:1278-1281. CrossRef Medline

Rick JM, Horschke I, Neuhauss SC (2000) Optokinetic behavior is reversed in achiasmatic mutant zebrafish larvae. Curr Biol 10:595-598. CrossRef Medline

Rinner O, Rick JM, Neuhauss SC (2005) Contrast sensitivity, spatial and temporal tuning of the larval zebrafish optokinetic response. Invest Ophthalmol Vis Sci 46:137-142. CrossRef Medline

Roeser T, Baier H (2003) Visuomotor behaviors in larval zebrafish after GFP-guided laser ablation of the optic tectum. J Neurosci 23:3726-3734. Medline

Sarvananthan N, Surendran M, Roberts EO, Jain S, Thomas S, Shah N, Proudlock FA, Thompson JR, McLean RJ, Degg C, Woodruff G, Gottlob I (2009) The prevalence of nystagmus: the Leicestershire nystagmus survey. Invest Ophthalmol Vis Sci 50:5201-5206. CrossRef Medline

Seth A, Culverwell J, Walkowicz M, Toro S, Rick JM, Neuhauss SC, Varga ZM, Karlstrom RO (2006) belladonna/(Ihx2) is required for neural patterning and midline axon guidance in the zebrafish forebrain. Development 133:725-735. CrossRef Medline

Shin YJ, Park KH, Hwang JM, Wee WR, Lee JH, Lee IB (2006) Objective measurement of visual acuity by optokinetic response determination in patients with ocular diseases. Am J Ophthalmol 141:327-332. CrossRef Medline

St John R, Fisk JD, Timney B, Goodale MA (1984) Eye movements of human albinos. Am J Optom Physiol Opt 61:377-385. CrossRef Medline

Straw AD (2008) Vision egg: an open-source library for realtime visual stimulus generation. Front Neuroinform 2:4. Medline

Tarpey P, et al. (2006) Mutations in FRMD7, a newly identified member of the FERM family, cause X-linked idiopathic congenital nystagmus. Nat Genet 38:1242-1244. CrossRef Medline

Thomas S, Proudlock FA, Sarvananthan N, Roberts EO, Awan M, McLean R, Surendran M, Kumar AS, Farooq SJ, Degg C, Gale RP, Reinecke RD, Woodruff G, Langmann A, Lindner S, Jain S, Tarpey P, Raymond FL, Gottlob I (2008) Phenotypical characteristics of idiopathic infantile nystagmus with and without mutations in FRMD7. Brain 131:1259-1267. Medline

Traber GL, Chen CC, Huang YY, Spoor M, Roos J, Frens MA, Straumann D, Grimm C (2012) Albino mice as an animal model for infantile nystagmus syndrome. Invest Ophthalmol Vis Sci 53:5737-5747. CrossRef Medline

Tusa RJ, Zee DS, Hain TC, Simonsz HJ (1992) Voluntary control of congenital nystagmus. Clin Vis Sci 7:195-210. 
Tusa RJ, Mustari MJ, Das VE, Boothe RG (2002) Animal models for visual deprivation-induced strabismus and nystagmus. Ann N Y Acad Sci 956: 346-360. CrossRef Medline

Watkins RJ, Thomas MG, Talbot CJ, Gottlob I, Shackleton S (2012) The Role of FRMD7 in Idiopathic Infantile Nystagmus. J Ophthalmol 2012: 460956. Medline
Wester ST, Rizzo JF 3rd, Balkwill MD, Wall C 3rd (2007) Optokinetic nystagmus as a measure of visual function in severely visually impaired patients. Invest Ophthalmol Vis Sci 48:4542-4548. CrossRef Medline

Yee RD, Baloh RW, Honrubia V (1980) Study of congenital nystagmus: optokinetic nystagmus. Br J Ophthalmol 64:926-932. CrossRef Medline 